\title{
O Iowa Gambling Task: Uma Revisão Crítica
}

\author{
Graça Areias ${ }^{1}$ \\ Rui Paixão \\ Ana Paula Couceiro Figueira \\ Universidade de Coimbra
}

\begin{abstract}
RESUMO - O Iowa Gambling Task (IGT) é uma tarefa amplamente utilizada na avaliação da capacidade de tomada de decisão. Neste artigo, procede-se à revisão da literatura, comparando-se as versões do IGT, as diferentes medidas de avaliação do desempenho e as alterações introduzidas nos procedimentos, nomeadamente no feedback, na aleatorização espacial dos baralhos, no número de ensaios e de cartas por baralho, nas instruções, na remuneração e na manipulação das recompensas e punições. Desta análise, conclui-se que as diversas versões da tarefa, as alterações nos procedimentos de aplicação e as diferentes medidas utilizadas na avaliação têm impacto no desempenho, prejudicam a comparação entre estudos e as generalizações dos resultados. Finalmente, apresentam-se sugestões para uma maior adequação dos procedimentos.
\end{abstract}

Palavras-chave: Iowa Gambling Task, tomada de decisão, feedback, recompensas, punições

\section{The Iowa Gambling Task: A Critical Revision}

\begin{abstract}
The Iowa Gambling Task (IGT) is a widely used task in the assessment of the decision-making ability. In this article, we conduct a literature review by comparing IGT versions, different performance assessment measures, and changes to procedures including feedback, spatial randomization of decks, number of trials, number of cards per deck, instructions, payment, and rewards and punishments. On basis of this analysis, we conclude that different versions of the task, changes in application procedures and different measures used to assess the task have an impact on performance, thereby affecting comparison among studies and generalization of results. Finally, we offer suggestions to define adequate procedures.
\end{abstract}

Keywords: Iowa Gambling Task, decision-making, feedback, rewards, punishments

O Iowa Gambling Task (IGT) é uma tarefa elaborada para simular situações da vida real de tomada de decisão em condições de incerteza, relativamente às consequências, ou seja, quanto à recompensa ou punição (Bechara, Damasio, Damasio, \& Anderson, 1994). Foi desenvolvido face à inexistência de uma prova capaz de avaliar o papel das emoções na capacidade de tomada de decisão em doentes com lesões no córtex orbitofrontal e sustenta a hipótese do marcador somático (Damásio, 1994/2003). Atualmente, reveste-se de particular importância em função do grande número de estudos com referência ou utilização do instrumento: uma pesquisa por "Iowa Gambling Task" no Google evidencia 1.550.000 entradas, na Online Knowledge Library (b-on) 1008 resultados e na Web of Knowledge (WOK) 530. Utilizando a designação alternativa de "Iowa Gambling Test" aparecem no Google 4.840 .000 entradas.

O IGT original (Bechara et al., 1994) é um jogo em que o sujeito tem de escolher cartas de quatro baralhos diferentes (A, B, C, D). O objetivo do jogo é ganhar o máximo dinheiro possível ou evitar perder, sendo atribuído ao sujeito um crédito inicial de 2.000 dólares. Sempre que o indivíduo seleciona uma carta recebe uma recompensa monetária, mas

1 Endereço para correspondência: Unidade de Desabituação de Coimbra, Instituto da Droga e da Toxicodependência - Morada: Pavilhão n. ${ }^{\circ} 12$ do Hospital Sobral Cid, Conraria. Coimbra, Portugal. Código Postal: 3040-714. E-mail: gracaareias@gmail.com. em algumas cartas a recompensa é seguida de perda variável de dinheiro. Cada baralho tem uma sequência pré-definida de recompensas e punições. O sujeito desconhece o número de cartas por baralho e o número de escolhas que tem de fazer (100 seleções).

Os baralhos A e B oferecem as recompensas mais altas (100 dólares a cada seleção), mas também as punições mais elevadas (1.250 dólares em cada 10 seleções). Relativamente à frequência das punições que se repetem em cada 10 seleções, no baralho B há apenas uma punição de 1.250 dólares, enquanto no baralho A há cinco punições de 150 , 200, 250, 300 e 350 dólares. Esses baralhos são classificados por Bechara et al. (1994) como "desvantajosos" porque, a longo prazo, as penalizações monetárias ultrapassam as recompensas. Os baralhos $\mathrm{C}$ e $\mathrm{D}$ proporcionam recompensas mais baixas (50 dólares a cada seleção), mas também produzem perdas significativamente mais leves (250 dólares em cada 10 ensaios). Relativamente à frequência das punições que se repetem em cada 10 seleções, no baralho D há uma punição de 250 dólares, e no baralho $\mathrm{C}$ há cinco punições de 25,50 e 75 dólares. São classificados por Bechara et al. (1994) como "vantajosos" porque, a longo prazo, as recompensas ultrapassam as penalizações. Assim, para conseguir ganhar dinheiro, que é o objetivo da tarefa, a estratégia mais eficaz é selecionar mais cartas dos baralhos $\mathrm{C}$ e $\mathrm{D}$, do que dos baralhos A e B. A apreensão cognitiva dessa estratégia só pode ser adquirida através da experiên- 
cia. Contudo, os sujeitos normais decidem vantajosamente antes de conhecerem a estratégia vantajosa, guiados pelos marcadores somáticos (sinais emocionais) que antecipam as emoções previamente desencadeadas pelas recompensas e punições associadas a cada baralho (Bechara, Damasio, Tranel, \& Damasio, 1997). O sucesso na realização da tarefa está, assim, dependente da articulação entre fatores emocionais e cognitivos.

Essa primeira versão, denominada versão original ABCD (Bechara et al., 1994), incluía um procedimento manual com cartas e dinheiro de imitação. Posteriormente, foi desenvolvida uma versão eletrónica com dinheiro virtual que manteve as mesmas pautas de reforço (Bechara, Damasio, Damasio, \& Lee, 1999). Em 2000, Bechara, Tranel e Damasio (2000) introduziram alterações nas pautas de reforço dando origem à versão eletrónica $\mathrm{A}^{\prime} \mathrm{B}^{\prime} \mathrm{C}^{\prime} \mathrm{D}$ ' e à versão modificada E'F`G'H’.

A versão final (Bechara, 2007) permite a alteração de várias características da tarefa antes da sua administração, tais como o tempo entre ensaios, o número de ensaios, o tipo de moeda e o saldo inicial. No entanto, as pautas de reforço da primeira versão (Bechara et al., 1994) têm sido as mais amplamente utilizadas.

A análise da literatura (Caroselli, Hiscock, Scheibel, \& Ingram, 2006) permite constatar que há alguns aspetos nas características da tarefa e nos procedimentos de aplicação que têm sido modificados e que parecem ter impacto no desempenho. As alterações, a seguir apresentadas, são relativas ao feedback, à aleatorização espacial dos baralhos, ao número de ensaios e cartas por baralho, às instruções, à utilização de remuneração e à manipulação do esquema de recompensas e punições.

Este trabalho tem por objetivo analisar o impacto da manipulação destas variáveis na tomada de decisão avaliada com o IGT. Foram realizadas pesquisas na b-on e $W O K$, tendo sido identificadas 402 referências, entre estudos que utilizavam o IGT para avaliação da tomada de decisão e trabalhos de revisão crítica sobre a tarefa. Desses foram selecionados para análise mais detalhada, por conterem informações pertinentes para o objetivo acima referido, 155 artigos com a utilização do IGT, 13 artigos de revisão crítica, sete teses de doutoramento, três capítulos de livros que analisam o instrumento e o manual do IGT.

\section{Variações sobre o feedback}

A versão eletrónica do IGT (Bechara et al., 1999) é a mais amplamente utilizada. Permite disponibilizar feedback numérico sobre as recompensas e punições, feedback visual sobre perdas e ganhos em cada jogada (é mostrado um happy smiley quando o saldo é positivo e um sad smiley quando o saldo é negativo) e a atualização do saldo a cada jogada.

No entanto, há estudos que se limitam a dar feedback do ganho ou perda da jogada e não fornecem feedback sobre o desempenho global (Garon \& Moore, 2004). A introdução dessa modificação, ao retirar informação sobre a atualização do saldo, necessária ao sucesso na tarefa, pode levar os sujeitos a escolher com base nas reações afetivas imediatas às perdas e ganhos.

\section{Aleatorização espacial dos baralhos}

A aleatorização da disposição espacial dos baralhos foi adotada por vários estudos (e.g. Cella, Dymond, Cooper, \& Turnbull, 2007; Chiu \& Lin, 2007; Crone \& van der Molen, 2004; Dretsch \& Tipples, 2008; Garon \& Moore, 2004; Geurts, van der Oord, \& Crone 2006; Lin, Chiu, Lee, \& Hsieh, 2007; Pecchinenda, Dretsch, \& Chapman, 2006). Outros autores, no entanto, não aleatorizam os baralhos (e.g. Davis, Patte, Tweed, \& Curtis, 2007; Wood, Busemeyer, Koling, Cox, \& Davis, 2005), alegando ausência de impacto significativo no desempenho do efeito de ordem ABCD (Stout, Rodawalt, \& Siemers, 2001).

No entanto, recomenda-se aleatorizar, porque "assumindo a adoção duma estratégia esquerda-direita, os baralhos de recompensa alta estão situados, espacialmente, antes dos baralhos vantajosos" (Hooper, Luciana, Conklin, \& Yarger, 2004, p. 1150). É particularmente importante a aleatorização em caso de reteste, porque a manutenção da mesma ordem permite o conhecimento das pautas de reforço dos baralhos, diminuindo o caráter de incerteza da tarefa.

\section{Variação no número de cartas por baralho e ensaios}

No que respeita ao número de cartas, na primeira versão (Bechara et al., 1994), cada baralho tem 40 cartas, o que implica que quando se esgotam as cartas de um baralho, o sujeito recebe instruções para escolher dos restantes (Bechara, et al., 1999). Essa ocorrência pode limitar as escolhas dos sujeitos e enviesar os resultados. Apesar dos autores (Bechara et al., 1999) referirem que essa situação é rara, a versão A 'B 'C 'D' (Bechara, Tranel, \& Damasio, 2000) passa a incluir 60 cartas por baralho.

Em relação ao número de ensaios, alguns estudos com crianças e adolescentes utilizam versões do IGT com 150 (Crone, Bunge, Latenstein, \& van der Molen, 2005) ou com 200 ensaios (e.g. Crone \& van der Molen, 2004; Crone, Vendel, \& van der Molen, 2003; Huizenga, Crone, \& Jansen, 2007; Overman, 2004; Overman et al., 2004), contrariamente aos clássicos 100 ensaios, por considerarem que os jovens necessitam de mais experiência para aprender (Overman et al., 2004).

É esperado que a alteração do número de ensaios modifique o desempenho, porque como os indivíduos só podem aprender com a experiência, as primeiras jogadas são, necessariamente, exploratórias (Dunn, Dalgleish, \& Lawrence, 2006; Maia \& McClelland, 2004; Monterosso, Ehrman, Napier, O’Brien, \& Childress, 2001).

$\mathrm{O}$ aumento do número de ensaios permite testar a hipótese dos ritmos de aprendizagem serem mais lentos nalguns grupos experimentais, o que reforça a importância da análise da evolução do desempenho. Outra alternativa, ainda não suficientemente explorada, que permite avaliar a aprendizagem, é a realização de reteste (e.g. Verdejo- 
-Garcia et al., 2007), que tem a vantagem de não introduzir modificações na tarefa.

\section{Tipo de instruções}

No estudo de Bechara et al. (1999) são introduzidas alterações nas instruções da tarefa original (Bechara et al., 1994), explicitando-se que há baralhos melhores e piores e que o experimentador não altera a ordem pré-definida das cartas. Isto porque, como refere Bechara et al. (2000), nos estudos iniciais os sujeitos pensavam que nunca podiam ganhar, porque o computador gerava esquemas de recompensa e punição, independentemente das escolhas. As publicações subsequentes adotam essas instruções detalhadas (e.g. Bechara, 2007; Bechara et al., 2000; Bechara \& Damasio, 2002; Bechara, Dolan, \& Hindes, 2002).

Schmitt, Brinkley e Newman (1999) não encontraram diferenças no desempenho entre psicopatas e grupo controlo com a utilização duma versão do IGT (Bechara et al., 1994) sem instruções detalhadas, tendo ambos os grupos apresentado um desempenho pouco vantajoso. Pelo contrário, Blair, Colledge e Mitchell (2001) utilizaram instruções detalhadas (Bechara et al., 1999), num estudo com crianças e adolescentes de 9 a 17 anos com tendências psicopatas, constatando que estes, contrariamente ao grupo controlo, não apresentaram desempenho vantajoso. Os autores colocaram a hipótese das instruções detalhadas serem responsáveis pelas diferenças entre os grupos, ausentes no estudo de Schmitt et al. (1999), o que parece ir ao encontro do observado por Mitchell, Colledge, Leonard e Blair (2002). Nesse estudo, também com indivíduos psicopatas, Mitchell et al. (2002) concluíram que as instruções detalhadas induzem, no grupo controlo, o desenvolvimento de preferência pelos baralhos vantajosos. No mesmo sentido, Balodis, MacDonald e Olmstead (2006) estudaram o impacto das instruções simplificadas (Bechara et al., 1994) versus detalhadas (Bechara et al., 1999) no desempenho no IGT, tendo constatado que os grupos que receberam as instruções detalhadas apresentaram um desempenho significativamente superior logo após os primeiros 20 ensaios.

O conhecimento do objetivo do estudo versus apresentação do IGT como tarefa distratora, foi outro aspeto da instrução alvo de manipulação, sendo que o grupo colocado na primeira condição apresentou um desempenho significativamente superior. Os autores (Balodis et al., 2006) concluíram que o desempenho no IGT é muito sensível às pistas fornecidas pelas instruções, sendo muito importante controlar essa variável e interpretar com cautela os resultados dos estudos que não especificam as instruções fornecidas.

Assim e apesar de alguns resultados contraditórios (Glicksohn \& Zilberman, 2010), a maioria dos estudos realça a importância da utilização de instruções detalhadas no desempenho na tarefa, sendo recomendável adotar a formulação de Bechara et al. (1999).

Outro aspeto relativo às instruções é o modo como são fornecidas, inicialmente de forma oral (Bechara et al., 1994; Bechara et al., 1999; Bechara et al., 2000), e na versão final escritas num cartão (Bechara, 2007). Noutros estudos, as instruções estão inseridas na aplicação eletrónica (e.g. Fernie \& Tunney, 2006; Stocco, Fum, \& Napoli, 2009; van den Bos, Houx, \& Spruijt, 2006). Pelo que nos é dado conhecer não foram realizados estudos comparativos entre as diferentes modalidades de administração das instruções. No entanto, vale destacar que a extensão das instruções detalhadas (Bechara et al., 1999) pode tornar difícil a sua compreensão, quando transmitidas de forma oral.

\section{Utilização de reforço}

Um aspeto que pode ter impacto na motivação e no desempenho é o facto de os sujeitos serem remunerados. Efetivamente, alguns estudos remuneram os participantes, independentemente do desempenho (e.g. Bechara, Damasio, Tranel \& Anderson, 1998; Bechara et al., 2000; Bechara et al., 2002; Bolla et al., 2003; Denburg, Recknor, Bechara, \& Tranel, 2006; Denburg, Tranel \& Bechara, 2005; Mazas, Finn, \& Steinmetz, 2000; Monterosso et al., 2001).

Outros estudos compararam o desempenho no IGT em função do tipo de reforço, ou seja, a utilização de dinheiro real versus dinheiro de imitação e, apesar de não terem encontrado diferenças significativas entre as condições (Bowman \& Turnbull, 2003; Carter \& Pasqualini, 2004), constataram que a utilização de dinheiro de imitação produz maior variabilidade nos resultados (Bowman \& Turnbull, 2003), por induzir um comportamento menos cauteloso (Evans, Kemish, \& Turnbull, 2004).

Outra condição que pode fomentar a motivação é condicionar a remuneração ao desempenho (e.g. Campbell, Stout, \& Finn, 2004; Davis et al., 2007; Petry, Bickel, \& Arnett, 1998; Suzuki, Hirota, Takasawa, \& Shigemasu, 2003). Diversos estudos (e.g. Bowman \& Turnbull, 2003; Carter \& Pasqualini, 2004; Hooper et al., 2004; Schmitt et al., 1999) alteraram o crédito inicial do IGT (Bechara et al., 1994) e adaptaram as recompensas e punições, mantendo a proporção de ganhos e perdas, de modo a que os participantes pudessem receber o saldo final em dinheiro real.

Curiosamente, os estudos de Schmitt et al. (1999) realizados com psicopatas, apesar de condicionarem a remuneração ao desempenho, não encontraram diferenças relativamente ao grupo controlo. Pelo contrário, Blair et al. (2001) e Mitchell et al. (2002) encontraram diferenças no mesmo tipo de população, não tendo oferecido qualquer tipo de remuneração, resultado que atribuíram à utilização de instruções explícitas, não utilizadas por Schmitt et al. (1999).

Fernie e Tunney (2006) compararam o efeito das instruções (sugestão de que há baralhos melhores e piores vs. sem sugestão) e do tipo de reforço (real vs. imitação), tendo concluído que o desempenho só é afetado pela utilização de reforço real quando não são dadas instruções detalhadas. Também Vadhan, Hart, Haney, van Gorp e Foltin (2009) confrontaram o desempenho no IGT em consumidores abusivos de cocaína nas duas condições de contingência monetária (real vs. imitação), tendo concluído que a utilização de reforço real melhora o desempenho dos consumidores abusivos de cocaína. Esses resultados divergem do estudo de Verdejo-Garcia et al. (2007) em que esses pacientes apresentam desempenho 
desvantajoso em ambas as condições. No entanto, Vadhan et al. (2009) atribuíram essas diferenças aos tempos de abstinência dos indivíduos: três dias de abstinência (Vadhan et al., 2009) versus 25 dias (Verdejo-Garcia et al., 2007). Esses estudos alertam para a existência de diferenças individuais na sensibilidade a contingências monetárias, sendo importante investigar o desempenho de alguns grupos clínicos.

\section{Manipulação das recompensas e punições}

A manipulação das recompensas e punições, no que respeita à frequência ou quantias, é a diferença mais significativa que se pode introduzir na tarefa e foi realizada para testar hipóteses. Neste sentido, van den Bos et al. (2006) estudaram o efeito das diferenças na grandeza da recompensa entre os baralhos desvantajosos (A/B) e vantajosos (C/D), mantendo a frequência e o saldo líquido em cada 10 jogadas. A alteração da proporção da recompensa de 2:1 (A/B:C/D, \$100:\$50) para 1:1 (\$50:\$50) diminui a diferença na grandeza da recompensa, sendo que as diferenças entre os baralhos só são determinadas pela punição. Essa condição leva a menos escolhas dos baralhos desvantajosos. Pelo contrário, quando a grandeza das diferenças aumenta para 4:1 (200:50) e 6:1 (300:50), os participantes tendem a escolher mais os baralhos desvantajosos. Esse estudo revela que o desempenho no IGT é sensível a diferenças na magnitude das recompensas imediatas entre os baralhos vantajosos e desvantajosos, ou seja, uma eventual preferência pelos baralhos A e B pode ser explicada pela magnitude das recompensas.

A versão A 'B ' C 'D ' do IGT (Bechara et al., 2000) foi desenvolvida para verificar se os pacientes com lesões no córtex orbitofrontal melhoravam o desempenho com o aumento das punições. É referido que essa versão é análoga à $\mathrm{ABCD}$ e que a única diferença é uma mudança na frequência ou quantia da punição em que a discrepância entre recompensa e punição nos baralhos desvantajosos (A`e B`) é maior em direção negativa, i.e., induz maiores perdas. Pelo contrário, nos baralhos vantajosos (C’ e D`), a discrepância entre recompensa e punição é maior em direção positiva, i.e., induz maiores ganhos. No entanto, não foram divulgados os valores das punições e recompensas.

A versão comercializada (Bechara, 2007), também denominada A 'B 'C'D', apresenta diferenças significativas, relativamente à versão original, no padrão das recompensas e das punições. Cada baralho tem 60 cartas organizadas em seis blocos de 10 cartas. As recompensas passam a ter valores variáveis e as quantias aumentam ao longo dos seis blocos. Assim, as recompensas das primeiras 10 seleções passam a ser em média 100 dólares nos baralhos A `e B`, e 50 dólares nos baralhos C' e D'. Nos blocos seguintes, as recompensas aumentam em média, nos baralhos A`e B`, 10 dólares e, nos baralhos $C^{\prime}$ e D', cinco dólares. No que respeita às punições, também há alterações nas quantias e na frequência ao longo dos seis blocos. No entanto, o montante total das punições é igual para os baralhos A ' e B' e C'e D'.

No baralho A', no primeiro bloco, há cinco punições imprevisíveis, que vão de 150 a 350 dólares, perfazendo uma perda total de 1.250 dólares. A frequência de punição aumenta uma carta por bloco, sendo que no segundo bloco há seis punições (entre 150 a 350 dólares), perfazendo um total de 1.500 dólares. O montante das punições agrava 250 dólares por bloco. A perda total para o baralho A` é de 3.750 dólares. No baralho B', a frequência da punição mantem-se constante ao longo dos blocos. Assim, no primeiro bloco há uma perda única total de 1.250 dólares, que vai aumentando 250 dólares por bloco perfazendo, tal como no baralho A', uma perda total de 3.750 dólares.

No que respeita à frequência das punições no baralho $C^{\prime}$, há cinco punições de 50 dólares no primeiro bloco (que perfazem uma quantia de 250 dólares) e aumentam uma punição por bloco, passando a ser seis no segundo bloco e assim sucessivamente, perfazendo uma perda total de 1.875 dólares. No baralho D', no primeiro bloco há uma perda única de 1.250 dólares, que vai aumentando 25 dólares por bloco, perfazendo, tal como no baralho $\mathrm{C}^{\prime}$, uma perda total de 1.875 dólares.

Vale destacar, que nos baralhos A' e C', o aumento da frequência das punições (uma carta por bloco) implica que no último bloco todas as escolhas estejam associadas a punições. Por outro lado, o facto dos valores das recompensas em todos os baralhos deixarem de ser fixos e passarem a ser valores médios, que aumentam ao longo dos blocos, dificulta o conhecimento do padrão de recompensa. No entanto, constata-se na versão A 'B 'C 'D' um aumento significativo nas diferenças entre baralhos vantajosos e desvantajosos, relativamente aos saldos líquidos totais. Assim, o saldo líquido total para os baralhos A e B, na versão ABCD, é de -1.000 dólares, enquanto na versão A 'B ' C 'D' é de -3.750 dólares. No que respeita aos baralhos $\mathrm{C}$ e $\mathrm{D}$, na versão $\mathrm{ABCD}$, o saldo líquido total é de 1.500 dólares, enquanto na versão A 'B 'C'D'é de 1.875 dólares. Efetivamente, os baralhos vantajosos tornam-se cada vez mais vantajosos e, inversamente, os baralhos desvantajosos cada vez mais desvantajosos (Bechara, 2007). Assim, se por um lado o conhecimento dos valores das recompensas parece ser dificultado pela inserção de valores médios, o agravamento das diferenças introduzidas nos saldos líquidos totais aponta precisamente no sentido contrário, ou seja, facilita o conhecimento dos baralhos vantajosos e desvantajosos. Contudo, pelo que nos é dado conhecer, não foram publicados estudos comparativos entre as versões $\mathrm{ABCD}$ e $\mathrm{A}^{\prime} \mathrm{B}^{\prime} \mathrm{C}^{\prime} \mathrm{D}$ ', nem explicitados os pressupostos que sustentaram essas modificações. Por este facto, não se conhece o impacto das diferenças introduzidas na tarefa na permeabilidade cognitiva das pautas de reforço.

\section{Avaliação de Desempenho no IGT}

A interpretação de resultados do IGT é condicionada pelas diversas medidas adotadas para avaliar o desempenho. Essa diversidade pode estar na origem de alguma divergência ou incoerência detetada nos resultados das investigações.

O desempenho foi inicialmente medido pela fórmula $(\mathrm{C}+$ D) - $(\mathrm{A}+\mathrm{B})$, que corresponde ao cálculo da diferença entre a soma total de escolhas dos baralhos vantajosos menos a soma total de escolhas dos baralhos desvantajosos (Bechara et al., 1994).

Posteriormente, foi definido como desempenho vantajoso $(\mathrm{C}+\mathrm{D})-(\mathrm{A}+\mathrm{B})>0$ e como desempenho desvantajoso $(\mathrm{C}+$ 
D) - (A + B) < 0 (Bechara et al., 1998; Bechara et al., 2000; Denburg et al., 2005), sendo a pontuação zero considerada um comportamento aleatório (Dalgleish et al., 2004; Denburg et al., 2006) e os valores que não se afastam significativamente dessa pontuação classificados como borderline (Denburg et al., 2006).

Nos trabalhos subsequentes (Bechara et al., 2002; Bechara \& Damasio, 2002), o valor 10 é adotado como ponto de corte, por ser a pontuação máxima alcançada pelos pacientes com lesões orbitofrontais (Bechara et al., 2001; Verdejo-Garcia, Aguilar de Arcos, \& Pérez-Garcia, 2004). Posteriormente, foi definido como desempenho desvantajoso uma pontuação inferior ou igual a -18 , como desempenho limítrofe uma pontuação entre -17 e 17, e como desempenho vantajoso uma pontuação superior ou igual a 18 (Bakos, Denburg, Fonseca, \& Parente, 2010).

Outra medida adotada para avaliar a evolução da aprendizagem (Bechara et al., 1999) é o cálculo por blocos, que consiste na divisão do desempenho em cinco fases de 20 ensaios, calculadas através da fórmula $(C+D)-(A+B)$. Enquanto o cálculo total mede o desempenho durante toda a tarefa, o cálculo por blocos permite analisar a evolução da aprendizagem, sendo considerado o índice mais sensível e específico do IGT (Weller, Levin, \& Bechara, 2010). Na comparação entre grupos, é indicado realizar-se, para além da análise intragrupos, uma análise por blocos intergrupos (Weller et al., 2010). Neste sentido, Dunn et al. (2006) sugerem a comparação relativamente ao comportamento aleatório, e alertam para a possibilidade das diferenças entre os grupos de pacientes e os controlos serem devidas ao ritmo de aprendizagem.

No que respeita à evolução da aprendizagem na tarefa, diversos estudos (Bechara et al., 1997; Bechara, 2007; Bechara, Damasio, Tranel, \& Damasio, 2005; Jollant et al., 2005; Oldershaw et al., 2009) identificaram a escolha 50 como o ponto médio no qual o grupo controlo começa a fazer mais escolhas vantajosas. Nesse contexto, partindo do princípio que as primeiras jogadas são exploratórias, alguns autores (Monterosso et al., 2001) recomendam que a análise se limite aos últimos 50 ensaios, porque estes são uma medida mais fiável do que o desempenho global.

Outro aspeto importante é a identificação de processos diferentes entre os primeiros e últimos ensaios da tarefa (Bechara, 2004; Brand, Labudda, \& Markowitsch, 2006; Noel, Bechara, Dan, Hanak, \& Verbanck, 2007), nomeadamente entre:

- Os primeiros 40 ensaios, em que as decisões são feitas sem conhecimento explícito das contingências de recompensa e punição que guiam a decisão na tarefa, baseando-se prioritariamente em processos implícitos, nomeadamente de aprendizagem afetiva;

- Os últimos 40 ensaios, em que é mais provável que os participantes tenham adquirido (pelo menos) conhecimento explícito sobre os riscos associados a cada baralho e as funções executivas tenham assumido um papel predominante.

Decorre, de acordo com os autores (Noel et al., 2007), uma mudança gradual na natureza da tarefa, ou seja, de tomada de decisão sob ambiguidade para tomada de decisão sob risco, sendo importante avaliar qual se encontra, ou não, deteriorada.
Outro aspeto que tem merecido bastante destaque na literatura é a negligência de outras diferenças entre os baralhos com base na distinção entre "vantajosos" e "desvantajosos" (Caroselli et al., 2006). Abordagens mais recentes (Bechara, 2007) sugerem que se analisem individualmente os quatro baralhos e compare o número de cartas selecionadas em cada um ao longo das cinco fases, dado que cada baralho parece apresentar caraterísticas específicas que escapam à análise tradicional.

Por exemplo, para além das diferenças na magnitude das recompensas e punições, os baralhos apresentam também diferenças na frequência das punições e das recompensas. No que respeita à frequência da punição, os baralhos $\mathrm{B}$ (“desvantajoso") e D ("vantajoso") apresentam frequência de punição baixa (ganho líquido em $90 \%$ dos ensaios), enquanto os A ("desvantajoso") e C ("vantajoso") são baralhos de frequência de punição alta (ganho líquido em $50 \%$ dos ensaios).

Alguns participantes parecem valorizar mais a frequência de resultados positivos do que as quantias de dinheiro (ganho ou perdido), por isso preferem os baralhos $\mathrm{B}$ e $\mathrm{D}$ (e.g. Bakos, Parente, \& Bertagnolli, 2010; Bark, Dieckmann, Bogerts, \& Northoff, 2005; Caroselli et al., 2006; Carvalho, 2010; Chiu \& Lin, 2007; Crone \& van der Molen, 2004; Garon \& Moore, 2004; Hooper et al., 2004; Huizenga et al., 2007; Lin et al., 2007; O'Carroll \& Papps, 2003; Overman, 2004; Ritter, Meador-Woodruf, \& Dalack, 2004; Schneider \& Parente, 2006; Toplak, Jain, \& Tannock, 2005; Wilder, Weinberger, $\&$ Goldberg, 1998).

Este facto vai ao encontro da importância atribuída à frequência, mais do que às quantias, na literatura de tomada de decisão (Cosmides \& Tooby, 1996; Gigerenzer \& Hoffrage, 1995; Slovic, Finucane, Peters, \& MacGregor, 2004). Por outro lado, também vai ao encontro dos estudos de reforço realizados em animais, em que se constata que a frequência (Schneider, 1973) e a previsibilidade da recompensa (Steinhauer, 1984) têm mais influência que a quantidade. Nomeadamente, a teoria do processamento de armazenamento automático da informação defende que a frequência de ocorrência de um acontecimento é um dos aspetos da experiência que é continuamente registrado na memória, independentemente da idade, aptidões, educação ou motivação de um indivíduo (Hasher \& Zacks, 1979, 1984; Zacks, Hasher, \& Sanft,1982). Também nesse sentido, Caroselli et al. (2006) verificam que o poder atrativo de um baralho depende da frequência prévia de reforço e não da magnitude da recompensa. Em conclusão, a frequência é uma dimensão dominante no IGT, sendo por isso recomendada a sua análise (Huizenga et al., 2007; Singh \& Khan, 2009; Schneider \& Parente, 2006; Wagner \& Parente, 2009).

Outro aspeto prende-se com a necessidade de redefinir o conceito de decisão vantajosa e a classificação dos baralhos como "vantajosos" ou "desvantajosos", porque no início da tarefa os sujeitos não conhecem as recompensas e punições associadas aos baralhos e porque os denominados "desvantajosos" podem ser muito atrativos nas primeiras jogadas e não oferecer nenhuma punição (Maia \& McClelland, 2004).

Nesse sentido, Wagar e Dixon (2006) diferenciam o desempenho ótimo (ou normativo) do desempenho vantajoso, referindo que o desempenho no IGT tem sido analisado do ponto de vista do experimentador, tendo por modelo o de- 
sempenho ótimo. Sugerem que a análise deve ser realizada tendo por referência o desempenho vantajoso, isto é, do ponto de vista dos participantes, o que implica avaliar cada seleção em função das escolhas que foram previamente realizadas. Por exemplo, no que respeita ao baralho B, Overman et al. (2006) referem que nas suas investigações e nos estudos de Bechara et al. (1994), a primeira punição é a nona carta do baralho. Dado que os participantes costumam explorar todos os baralhos, essa carta é encontrada no ensaio 25-30 nos estudos de Overman et al. (2006), enquanto em Bechara et al. (1997), essa carta é encontrada em média no ensaio 36.

\section{Discussão}

O grande número de estudos realizados com o IGT tem contribuído para o conhecimento da tarefa e dos mecanismos implicados na tomada de decisão. No entanto, a utilização de diferentes versões e a grande variabilidade nas características e procedimentos de aplicação afetam o desempenho e prejudicam a possibilidade de comparação entre estudos e a generalização dos resultados.

As diferentes versões e procedimentos de aplicação variam no que respeita ao padrão das recompensas e punições, ao feedback fornecido, à aleatorização espacial dos baralhos, a variação no número de cartas por baralho e ensaios, às instruções e à utilização de remuneração. Verifica-se como ponto fraco de alguns estudos o facto de não especificarem em detalhe todas as características e procedimentos experimentais adotados.

No que respeita às versões, as pautas de reforço do IGT de Bechara et al. (1994) são as mais frequentemente utilizadas, na versão eletrónica (Bechara et al., 1999). Essa versão tem a vantagem de permitir ofeedback sobre o desempenho global, facilitando a aprendizagem. A aleatorização espacial dos baralhos, particularmente em caso de reteste, surge também como um aspeto a considerar, evitando assim o efeito de ordem ABCD provocado pelos baralhos de recompensa alta estarem situados espacialmente antes dos baralhos de recompensa baixa. No que respeita ao número de ensaios, é importante manter as 100 seleções e aumentar para 100 o número de cartas por baralho, de forma a não limitar as escolhas dos sujeitos.

A tarefa parece ser bastante sensível às instruções adotadas, o que não é surpreendente numa tarefa concebida para avaliar o papel da emoção na tomada de decisão. Verifica-se que a utilização de instruções detalhadas (Bechara et. al, 1999), em que se refere que há baralhos melhores e piores e que o experimentador não altera a ordem pré-definida das cartas, parece favorecer a aprendizagem nos grupos controlo. O conhecimento do objetivo da tarefa, ou seja, de tomada de decisão, também se revelou como potenciador da aprendizagem. Com a exceção de alguns estudos que se dedicam a analisar o impacto das instruções, um largo número de trabalhos menospreza essa caraterística, não referindo quais são as instruções fornecidas. Relativamente à forma como são transmitidas, considera-se preferível a leitura das instruções pelos sujeitos em detrimento da transmissão oral.

No que respeita à remuneração, a situação ideal para avaliar o estilo de tomada de decisão exigiria que os indiví- duos investissem o seu dinheiro e efetivamente ganhassem e perdessem. Essa simulação da vida real não é possível realizar por motivos éticos e por questões de ordem prática e motivacional. Assim, quando os participantes estão a jogar condicionados pela remuneração, estão sempre no âmbito do ganho, porque efetivamente por mais que percam, do ponto de vista ético, nenhuma situação experimental permite que saiam da situação a perder dinheiro, ou seja, o resultado nunca será efetivamente negativo. Assim, parece que as condições que, aparentemente, aproximam a situação IGT duma situação de vida real, ou seja, a remuneração condicionada ao resultado do jogo, parecem de alguma forma perverter a natureza da situação, porque colocam os sujeitos na perspetiva de ganhar, mas nunca numa situação de perda real. Além disso, de acordo com a prospect theory (Kahneman \& Tversky, 1979), quando o ponto de referência é definido de tal forma que o resultado é visto como ganho, os indivíduos tendem a ser adversos ao risco. Desse ponto de vista, parece que condicionar a remuneração ao desempenho pode induzir um desempenho vantajoso. Aliás, a questão da motivação individual na participação nos estudos e o impacto nos resultados revela-se como um denominador comum a toda a investigação. Há dimensões, tais como a avaliação da personalidade, em que o facto de não haver boas ou más respostas não impede o fenómeno da desejabilidade social. No que respeita à avaliação de aptidões e competências, pressupõe-se que os sentimentos de sucesso ou fracasso sejam em si fatores motivadores. Seria ético remunerar um sujeito em função dos seus resultados na Escala de Inteligência de Wechsler para fomentar a motivação para participar?

Resta ainda a questão da sensibilidade individual à remuneração, dado que para alguns indivíduos esse fator é mais motivador do que para outros. Assim, perante estas questões, a alternativa de usar dinheiro virtual e não condicionar a remuneração ao desempenho revela-se um procedimento mais adequado.

No que respeita à manipulação das recompensas e punições, considera-se que qualquer alteração deve ser feita para testar determinadas hipóteses e sempre em comparação com a versão que é alvo de modificações. As alterações introduzidas na versão do IGT de Bechara (2007) parecem alterar de forma significativa a tarefa, sem terem sido publicados estudos comparativos.

Finalmente, um aspeto fundamental na análise de resultados diz respeito às medidas utilizadas para avaliar o desempenho. Por questões de comparação entre estudos, parece ser essencial utilizar a medida clássica, isto é, a fórmula $(\mathrm{C}+\mathrm{D})-(\mathrm{A}+\mathrm{B})$. No entanto, dado que essa medida se revelou pouco discriminatória dos diferentes processos de tomada de decisão em jogo ao longo da tarefa, torna-se também importante avaliar os ritmos e a capacidade de aprendizagem com base na evolução do desempenho por blocos, através dessa fórmula e na comparação entre grupos realizar uma análise por blocos intergrupos (Weller et al., 2010). Adotando diferentes metodologias, é possível compreender o estilo de tomada de decisão dos sujeitos, ir além da simplificação da divisão dos baralhos em bons e maus, e da classificação em desempenho vantajoso, desvantajoso e limítrofe, evidenciada pela fórmula clássica e analisar individualmente a evolução das escolhas. Uma 
análise separada das escolhas nos quatro baralhos permite evidenciar como os sujeitos lidam com as recompensas ou punições e como encaram a dimensão frequência de punição, identificando perfis específicos de desempenho, embora os estudos realizados com populações clínicas não tenham explorado comparativamente esses aspetos.

\section{Referências}

Bakos, D. S., Denburg, N., Fonseca, R. P., \& Parente, M. A. d. M. P. (2010). A cultural study on decision making: performance differences on the Iowa Gambling Task between selected groups of Brazilians and Americans. Psychology \& Neuroscience, 3(1), 101-107. doi: 10.3922/j.psns.2010.1.013

Bakos, D. S., Parente, M. A. d. M. P., \& Bertagnolli, A. C. (2010). A tomada de decisão em adultos jovens e em adultos idosos: Um estudo comparativo. Psicologia, Ciência e Profissão, 30(1), 162-173. Retrieved from http://www.scielo.br/scielo. php?script=sci_serial\&lng=pt\&pid=1414-9893

Balodis, I. M., MacDonald, T. K., \& Olmstead, M. C. (2006). Instructional cues modify performance on the Iowa Gambling Task. Brain and Cognition, 60(2), 109-117. doi: 10.1016/j. bandc.2005.05.007

Bark, R., Dieckmann, S., Bogerts, B., \& Northoff, G. (2005). Deficit in decision making in catatonic schizophrenia: An exploratory study. Psychiatry Research, 134(2), 131-141. doi: 10.1016/j. psychres.2004.04.013

Bechara, A. (2004). The role of emotion in decision-making: Evidence from neurological patients with orbitofrontal damage. Brain and Cognition, 55(1), 30-40. doi: 10.1016/j. bandc.2003.04.001

Bechara, A. (2007). Iowa Gambling Task. Professional Manual. Florida: Psychological Assessment Resources.

Bechara, A., \& Damasio, H. (2002). Decision-making and addiction (part I): Impaired activation of somatic states in substance dependent individuals when pondering decisions with negative future consequences. Neuropsychologia, 40(10), 1675-1689. doi:10.1016/S0028-3932(02)00015-5

Bechara, A., Damasio, A. R., Damasio, H., \& Anderson, S. W. (1994). Insensitivity to future consequences following damage to human prefrontal cortex. Cognition, 50, 7-15. doi: 10.1016/0010-0277(94)90018-3

Bechara, A., Damasio, H., Damasio, A. R., \& Lee, G. P. (1999). Different contributions of the human amygdala and ventromedial prefrontal cortex to decision-making. The Journal of Neuroscience, 19(13), 5473-5481. Retrieved from http:// www.jneurosci.org/

Bechara, A., Damasio, H., Tranel, D., \& Anderson, S. W. (1998). Dissociation of working memory from decision making within the human prefrontal cortex. The Journal of Neuroscience, 18(1), 428-437. Retrieved from http://www.jneurosci.org/

Bechara, A., Damasio, H., Tranel, D., \& Damasio, A. R. (1997). Deciding advantageously before knowing the advantageous strategy. Science, 275(5304), 1293-1295. doi: 10.1126/ science.275.5304.1293

Bechara, A., Damasio, H., Tranel, D., \& Damasio, A. R. (2005). The Iowa Gambling Task and the somatic marker hypothesis: Some questions and answers. Trends in Cognitive Sciences, 9(4), 159-162; discussion 162-154. doi: 10.1016/j.tics.2005.02.002
Bechara, A., Dolan, S., \& Hindes, A. (2002). Decision-making and addiction (part II): Myopia for the future or hypersensitivity to reward? Neuropsychologia, 40(10), 1690-1705. doi: 10.1016/ S0028-3932(02)00016-7

Bechara, A., Dolan, S., Denburg, N., Hindes, A., Anderson, S. W., $\&$ Nathan, P. E. (2001). Decision-making deficits, linked to a dysfunctional ventromedial prefrontal cortex, revealed in alcohol and stimulant abusers. Neuropsychologia, 39(4), 376389. doi: 10.1016/S0028-3932(00)00136-6

Bechara, A., Tranel, D., \& Damasio, H. (2000). Characterization of the decision-making deficit of patients with ventromedial prefrontal cortex lesions. Brain, 123(11), 2189-2202. doi: 10.1093/brain/123.11.2189

Blair, R., Colledge, E., \& Mitchell, D. (2001). Somatic markers and response reversal: Is there orbitofrontal cortex dysfunction in boys with psychopathic tendencies? Journal of Abnormal Child Psychology, 29(6), 499-511. doi: 10.1023/A:1012277125119

Bolla, K. I., Eldreth, D. A., London, E. D., Kiehl, K. A., Mouratidis, M., Contoreggi, C., . . . Ernst, M. (2003). Orbitofrontal cortex dysfunction in abstinent cocaine abusers performing a decisionmaking task. Neuroimage, 19(3), 1085-1094. doi: 10.1016/ S1053-8119(03)00113-7

Bos, R. van den, Houx, B. B., \& Spruijt, B. M. (2006). The effect of reward magnitude differences on choosing disadvantageous decks in the Iowa Gambling Task. Biological Psychology, 71(2), 155-161. doi: 10.1016/j.biopsycho.2005.05.003

Bowman, C. H., \& Turnbull, O. H. (2003). Real versus facsimile reinforcers on the Iowa Gambling Task. Brain and Cognition, 53(2), 207-210. doi: 10.1016/s0278-2626(03)00111-8

Brand, M., Labudda, K., \& Markowitsch, H. J. (2006). Neuropsychological correlates of decision-making in ambiguous and risky situations. Neural Networks, 19(8), 12661276. doi: 10.1016/j.neunet.2006.03.001

Campbell, M. C., Stout, J. C., \& Finn, P. R. (2004). Reduced autonomic responsiveness to Gambling Task losses in Huntington's disease. Journal of the International Neuropsychological Society, 10(2), 239-245. doi: 10.1017/ S1355617704102105

Caroselli, J. S., Hiscock, M., Scheibel, R. S., \& Ingram, F. (2006). The Simulated Gambling Paradigm applied to young adults: An examination of University students' performance. Applied Neuropsychology, 13(4), 203-212. doi: 10.1207/ s15324826an1304_1

Carter, S., \& Pasqualini, M. S. (2004). Stronger autonomic response accompanies better learning: A test of Damasio's somatic marker hypothesis. Cognition \& Emotion, 18(7), 901-911. doi: $10.1080 / 02699930341000338$

Carvalho, J. C. N. (2010). Tomada de decisão no Iowa Gambling Task: Estudos comparativos quanto às varáveis idade e escolaridade e estudo correlacional. Dissertação de Mestrado, Universidade Católica do Rio Grande do Sul, Porto Alegre, Brasil. Retrieved from http://tede.pucrs.br/tde_busca/arquivo. php? codArquivo $=2765$

Cella, M., Dymond, S., Cooper, A., \& Turnbull, O. (2007). Effects of decision-phase time constraints on emotion-based learning in the Iowa Gambling Task. Brain and Cognition, 64(2), 164169. doi: 10.1016/j.bandc.2007.02.003

Chiu, Y. C., \& Lin, C. H. (2007). Is deck C an advantageous deck in the Iowa Gambling Task? Behavioral and Brain Functions, 3(37), 1-11. doi: 10.1186/1744-9081-3-37 
Cosmides, L., \& Tooby, J. (1996). Are humans good intuitive statisticians after all? Rethinking some conclusions from the literature on judgment under uncertainty. Cognition, 58, 1-73. doi: 10.1016/0010-0277(95)00664-8

Crone, E. A., \& van der Molen, M. W. (2004). Developmental changes in real life decision making: Performance on a gambling task previously shown to depend on the ventromedial prefrontal cortex. Developmental Neuropsychology, 25(3), 251-279. doi: 10.1207/s15326942dn2503_2

Crone, E. A., Bunge, S. A., Latenstein, H., \& van der Molen, M. W. (2005). Characterization of children's decision making: Sensitivity to punishment frequency, not task complexity. Child Neuropsychology, 11(3), 245-263. doi: 10.1080/092970490911261

Crone, E. A., Vendel, I., \& van der Molen, M. W. (2003). Decisionmaking in disinhibited adolescents and adults: Insensitivity to future consequences or driven by immediate reward? Personality and Individual Differences, 35(7), 1625-1641. doi: 10.1016/s0191-8869(02)00386-0

Dalgleish, T., Yiend, J., Bramham, J., Teasdale, J. D., Ogilvie, A. D., Malhi, G., \& Howard, R. (2004). Neuropsychological processing associated with recovery from depression after stereotactic subcaudate tractotomy. The American Journal of Psychiatry, 161(10), 1913-1916. Retrieved from http://ajp. psychiatryonline.org/

Damásio, A. (2003). O erro de Descartes. Emoção, razão e cérebro humano (D. Vicente \& G. Segurado, Trad.). Mem Martins, Portugal: Publicações Europa América (Trabalho original publicado em 1994)

Davis, C., Patte, K., Tweed, S., \& Curtis, C. (2007). Personality traits associated with decision-making deficits. Personality and Individual Differences, 42(2), 279-290. doi: 10.1016/j. paid.2006.07.006

Denburg, N. L., Recknor, E. C., Bechara, A., \& Tranel, D. (2006). Psychophysiological anticipation of positive outcomes promotes advantageous decision-making in normal older persons. International Journal of Psychophysiology, 61(1), 19-25. doi: 10.1016/j.ijpsycho.2005.10.021

Denburg, N. L., Tranel, D., \& Bechara, A. (2005). The ability to decide advantageously declines prematurely in some normal older persons. Neuropsychologia, 43(7), 1099-1106. doi: 10.1016/j.neuropsychologia.2004.09.012

Dretsch, M. N., \& Tipples, J. (2008). Working memory involved in predicting future outcomes based on past experiences. Brain and Cognition, 66(1), 83-90. doi: 10.1016/j.bandc.2007.05.006

Dunn, B. D., Dalgleish, T., \& Lawrence, A. D. (2006). The somatic marker hypothesis: A critical evaluation. Neuroscience \& Biobehavioral Reviews, 30(2), 239-271. doi: 10.1016/j. neubiorev.2005.07.001

Evans, C. E. Y., Kemish, K., \& Turnbull, O. H. (2004). Paradoxical effects of education on the Iowa Gambling Task. Brain and Cognition, 54, 240-244. doi:10.1016/j.bandc.2004.02.022
Fernie, G., \& Tunney, R. J. (2006). Some decks are better than others: The effect of reinforcer type and task instructions on learning in the Iowa Gambling Task. Brain and Cognition, 60(1), 94-102. doi: 10.1016/j.bandc.2005.09.011

Garon, N., \& Moore, C. (2004). Complex decision-making in early childhood. Brain and Cognition, 55(1), 158-170. doi: 10.1016/ S0278-2626(03)00272-0

Geurts, H. M., Oord, S. van der, \& Crone, E. A. (2006). Hot and cool aspects of cognitive control in children with ADHD: Decision-making and inhibition. Journal of Abnormal Child Psychology, 34(6), 813-824. doi: 10.1007/s10802-006-9059-2

Gigerenzer, G., \& Hoffrage, U. (1995). How to improve Bayesian reasoning without instruction: Frequency formats. Psychological Review, 102(4), 684-704. doi: 10.1037/0033295X.102.4.684

Glicksohn, J., \& Zilberman, N. (2010). Gambling on individual differences in decision making. Personality and Individual Differences, 48(5), 557-562. doi: 10.1016/j.paid.2009.12.006

Hasher, L., \& Zacks, R. T. (1979). Automatic and effortful processes in memory. Journal of Experimental Psychology: General, 108(3), 356-388. doi: 10.1037/0096-3445.108.3.356

Hasher, L., \& Zacks, R. T. (1984). Automatic processing of fundamental information - The case of frequency of occurrence. American Psychological Association, 39(12), 1372-1388. Retirado de http://www.psych.utoronto.ca/users/ hasherlab/

Hooper, C. J., Luciana, M., Conklin, H. M., \& Yarger, R. S. (2004). Adolescents' performance on the Iowa Gambling Task: Implications for the development of decision making and ventromedial prefrontal cortex. Developmental Psychology, 40(6), 1148-1158. doi: 10.1037/0012-1649.40.6.1148

Huizenga, H. M., Crone, E. A., \& Jansen, B. J. (2007). Decisionmaking in healthy children, adolescents and adults explained by the use of increasingly complex proportional reasoning rules. Developmental Science, 10(6), 814-825. doi: 10.1111/j.14677687.2007.00621.x

Jollant, F., Bellivier, F., Leboyer, M., Astruc, B., Torres, S., Verdier, R., . . Courtet, P. (2005). Impaired decision making in suicide attempters. American Journal of Psychiatry, 162(2), 304-310. Retrieved from http://ajp.psychiatryonline.org

Kahneman, D., \& Tversky, A. (1979). Prospect theory: An analysis of decision under risk. Econometrica, 47(2), 263291. Retrieved from http://www.pattonfunds.com/EDGE/ EDGE_pdfs/ProspectTheory.pdf

Lin, C. H., Chiu, Y. C., Lee, P. L., \& Hsieh, J. C. (2007). Is deck B a disadvantageous deck in the Iowa Gambling Task? Behavioral and Brain Functions, 3(16), 1-10. doi: 10.1186/1744-9081-3-16

Maia, T. V., \& McClelland, J. L. (2004). A reexamination of the evidence for the somatic marker hypothesis: What participants really know in the Iowa Gambling Task. Proceedings of the National Academy of Sciences of the United States of America 101(45), 16075-16080. doi: 10.1073/pnas.0406666101 
Mazas, C. A., Finn, P. R., \& Steinmetz, J. E. (2000). Decisionmaking biases, antisocial personality, and early-onset alcoholism. Alcoholism: Clinical and Experimental Research, 24(7), 1036-1040. doi: 10.1111/j.1530-0277.2000.tb04647.x

Mitchell, D. G. V., Colledge, E., Leonard, A., \& Blair, R. J. R. (2002). Risky decisions and response reversal: Is there evidence of orbitofrontal cortex dysfunction in psychopathic individuals? Neuropsychologia, 40(12), 2013-2022. doi: 10.1016/s0028-3932(02)00056-8

Monterosso, J., Ehrman, R., Napier, K. L., O’Brien, C. P., \& Childress, A. R. (2001). Three decision-making tasks in cocaine-dependent patients: Do they measure the same construct? Addiction, 96(12), 1825-1837. doi: 10.1046/j.13600443.2001.9612182512.x

Noel, X., Bechara, A., Dan, B., Hanak, C., \& Verbanck, P. (2007). Response inhibition deficit is involved in poor decision making under risk in nonamnesic individuals with alcoholism. Neuropsychology, 21(6), 778-786. doi: 10.1037/08944105.21.6.778

O’Carroll, R. E., \& Papps, B. P. (2003). Decision making in humans: The effect of manipulating the central noradrenergic system. Journal of Neurology, Neurosurgery \& Psychiatry, 74, 376-378. doi: 10.1136/jnnp.74.3.376

Oldershaw, A., Grima, E., Jollant, F., Richards, C., Simic, M., Taylor, L., \& Schmidt, U. (2009). Decision making and problem solving in adolescents who deliberately self-harm. Psychological Medicine, 39(1), 95-104. doi: 10.1017/ S0033291708003693

Overman, W. H. (2004). Sex differences in early childhood, adolescence, and adulthood on cognitive tasks that rely on orbital prefrontal cortex. Brain and Cognition, 55(1), 134-147. doi: 10.1016/S0278-2626(03)00279-3

Overman, W. H., Frassrand, K., Ansel, S., Trawalter, S., Bies, B., \& Redmond, A. (2004). Performance on the Iowa Card Task by adolescents and adults. Neuropsychologia, 42(13), 1838-1851. doi: 10.1016/j.neuropsychologia.2004.03.014

Overman, W. H., Graham, L., Redmond, A., Eubank, R., Boettcher, L., Samplawski, O., \& Walsh, K. (2006). Contemplation of moral dilemmas eliminates sex differences on the Iowa Gambling Task. Behavioral Neuroscience, 120(4), 817-825. doi: 10.1037/0735-7044 .120.4.817

Pecchinenda, A., Dretsch, M., \& Chapman, P. (2006). Working memory involvement in emotion-based processes underlying choosing advantageously. Experimental Psychology, 53(3), 191-197. doi: 10.1027/1618-3169.53.3.191

Petry, N. M., Bickel, W. K., \& Arnett, M. (1998). Shortened time horizons and insensitivity to future consequences in heroin addicts. Addiction, 93(5), 729-738. doi: 10.1046/j.13600443.1998.9357298.x
Ritter, L. M., Meador-Woodruff, J. H., \& Dalack, G. W. (2004). Neurocognitive measures of prefrontal cortical dysfunction in schizophrenia. Schizophrenia Research, 68(1), 65-73. doi: 10.1016/S0920-9964(03)00086-0

Schmitt, W. A., Brinkley, C. A., \& Newman, J. P. (1999). Testing Damasio's somatic marker hypothesis with psychopathic individuals: Risk takers or risk averse? Journal of Abnormal Psychology, 108(3), 538-543. Retrieved from http://www.apa. org/pubs/journals/abn/index.aspx

Schneider, D. D. G., \& Parente, M. A. d. M. P. (2006). O desempenho de adultos jovens e idosos na Iowa Gambling Task (IGT): Um estudo sobre a tomada de decisão. Psicologia: Reflexão $e$ Crítica, 19, 442-450. doi: 10.1590/S0102-79722006000300013

Schneider, J. W. (1973). Reinforcer effectiveness as a function of reinforcer rate and magnitude: A comparison of concurrent performances. Journal of the Experimental Analysis of Behavior, 20(3), 461-471. doi: 10.1901/jeab.1973.20-461

Singh, V., \& Khan, A. (2009). Heterogeneity in choices on Iowa Gambling Task: Preference for infrequent-high magnitude punishment. Mind \& Society, 8(1), 43-57. doi: 10.1007/ s11299-008-0050-1

Slovic, P., Finucane, M. L., Peters, E., \& MacGregor, D. G. (2004). Risk as analysis and risk as feelings: Some thoughts about affect, reason, risk, and rationality. Risk Analysis, 24(2), 311322. doi: 10.1111/j.0272-4332.2004.00433.x

Steinhauer, G. D. (1984). Preference for predictacle small rewards over unpredictable larger rewards. Psychological Reports, 54(2), 467-471. doi: 10.2466/pr0.1984.54.2.467

Stocco, A., Fum, D., \& Napoli, A. (2009). Dissociable processes underlying decisions in the Iowa Gambling Task: A new integrative framework. Behavioral and Brain Functions, 5(1), 1-12. doi: 10.1186/1744-9081-5-1

Stout, J. C., Rodawalt, W. C., \& Siemers, E. R. (2001). Risky decision making in Huntington's disease. Journal of the International Neuropsychological Society, 7(01), 92-101. doi: 10.1017/S1355617701711095

Suzuki, A., Hirota, A., Takasawa, N., \& Shigemasu, K. (2003). Application of the somatic marker hypothesis to individual differences in decision making. Biological Psychology, 65(1), 81-88. doi: 10.1016/S0301-0511(03)00093-0

Toplak, M. E., Jain, U., \& Tannock, R. (2005). Executive and motivational processes in adolescents with Attention-DeficitHyperactivity Disorder (ADHD). Behavioral and Brain Function, 1(8), 1-12. doi: 10.1186/1744-9081-1-8

Vadhan, N. P., Hart, C. L., Haney, M., van Gorp, W. G., \& Foltin, R. W. (2009). Decision-making in long-term cocaine users: Effects of a cash monetary contingency on Gambling Task performance. Drug and Alcohol Dependence, 102(1-3), 95101. doi: 10.1016/j.drugalcdep.2009.02.003 
Verdejo-Garcia, A., Aguillar de Arcos, F., \& Pérez-Garcia, M. (2004). Alteraciones de los procesos de toma de decisiones vinculados al córtex prefrontal ventromedial en pacientes drogodependientes. Revista de Neurologia, 38(7), 601-606. Retrieved from http:/www.revneurol.com/

Verdejo-Garcia, A., Benbrook, A., Funderburk, F., David, P., Cadet, J. L., \& Bolla, K. I. (2007). The differential relationship between cocaine use and marijuana use on decision-making performance over repeat testing with the Iowa Gambling Task. Drug and Alcohol Dependence, 90(1), 2-11. doi: 10.1016/j. drugalcdep.2007.02.004

Wagar, B. M., \& Dixon, M. (2006). Affective guidance in the Iowa Gambling Task. Cognitive, Affective, Behavioral Neuroscience, 6(4), 277-290. Retrieved from http://www.springerlink.com/ content/1530-7026/

Wagner, G. P., \& Parente, M. A. d. M. P. (2009). O desempenho de idosos quanto a tomada de decisão em duas variáveis do Iowa Gambling Test. Psicologia: Teoria e Pesquisa, 25(3), 425-433. doi: 10.1590/S0102-37722009000300017

Weller, J. A., Levin, I. P., \& Bechara, A. (2010). Do individual differences in Iowa Gambling Task performance predict adaptive decision making for risky gains and losses? Journal of Clinical \& Experimental Neuropsychology, 32(2), 141-150. doi: 10.1080/13803390902881926
Wilder, K. E., Weinberger, D. R., \& Goldberg, T. E. (1998). Operant conditioning and the orbitofrontal cortex in schizophrenic patients: Unexpected evidence for intact functioning. Schizophrenia Research, 30(2), 169-174. doi: 10.1016/s09209964(97)00135-7

Wood, S., Busemeyer, J., Koling, A., Cox, C., \& Davis, H. (2005). Older adults as adaptive decision-makers: Evidence from the Iowa Gambling Task. Psychology and Aging, 20(2), 220-225. doi: 10.1037/0882-7974.20.2.220

Zacks, R. T., Hasher, L., \& Sanft, H. (1982). Automatic encoding of event frequency: Further findings. Journal of Experimental Psychology: Learning, Memory, and Cognition, 8(2), 106-116. Retrieved from http://www.psych.utoronto.ca/users/hasherlab/ publications.htm
Recebido em 12.01.2012

Primeira decisão editorial em 16.11.2012

Versão final em 11.12.2012

Aceito em 11.01.2013

\section{$2^{\circ}$ CONGRESSO DE QUALIDADE DE VIDA NO TRABALHO NO SERVIÇO PÚBLICO}

Período: 28 a 30 de agosto

Local: Brasília, DF 